\title{
AML1-ETO-positive acute myeloid leukemia presents different phenotype in pediatric and adult patients
}

\author{
Guopan $\mathrm{Yu}^{1}$, Zhao Yin ${ }^{1}$, Ying Yang ${ }^{1}$, Jiaheng Zhou ${ }^{2}$, Jiale Qiu ${ }^{2}$, Jinchang Zhou ${ }^{2}$, \\ Changxin Yin ${ }^{1}$, Dan Fang ${ }^{1}$, Xiaolan Xu${ }^{1}$, Xuejie Jiang ${ }^{1}$, Qifa Liu ${ }^{1}$, Danian $\mathrm{Nie}^{3}$, \\ Shuangfeng Xie ${ }^{3}$, Yiqing $\mathrm{Li}^{3}$, Sanfang Tu${ }^{4}$, Zhenqian Huang ${ }^{5}$, HUI ZHANG ${ }^{6}$, and Dan Xu${ }^{1}$ \\ ${ }^{1}$ Southern Medical University Nanfang Hospital \\ ${ }^{2}$ Southern Medical University \\ ${ }^{3}$ Sun Yat-Sen Memorial Hospital \\ ${ }^{4}$ Zhujiang Hospital, Southern Medical University \\ ${ }^{5}$ The First Affiliated Hospital of Guangzhou Medical University \\ ${ }^{6}$ Guangzhou Women and Children's Medical Center
}

October 8, 2020

\begin{abstract}
Background: C-KIT mutation and extramedullary infiltration highly occur in AML1-ETO-positive acute myeloid leukemia (AE-AML). Whether they having the same clinical significance in pediatric and adult patients remains unsure. Procedure: Totally 75 pediatric and 98 adult patients with newly diagnosed AE-AML from 5 centers were included and analyzed. Results: The incidence of extramedullary leukemia (EML) $(13.3 \%$ vs. $29.6 \%, \mathrm{P}=0.008)$ and c-KIT mutations $(12 / 68,17.6 \%$ vs. $30 / 74$, $40.5 \%, \mathrm{P}=0.003)$ in pediatric patients were less than half compared to adult counterpart. The disease outcome including complete response (CR), disease-free survival (DFS) and overall survival (OS) was comparable between the two patient groups, when induction/consolidation regimens were taken into consideration. Based on multivariate analysis, EML was associated with a higher relapse and worse survival, and patients carrying c-KIT mutations showed a trend toward a worse prognosis. Grouping by age, the adverse effect of EML and c-KIT mutations on prognosis was only seen in adults but not children and was not affected by consolidation regimens. Conclusions: The occurrence of EML and c-KIT mutations was much less in pediatric than adult AE-AML. Both EML and c-KIT mutations adversely impacted the disease outcome in adult but not pediatric patients, suggesting the behavior of AML1-ETO fusion protein might be age-dependent.
\end{abstract}

\section{Abbreviations}

AE-AML, AML1-ETO-positive acute myeloid leukemia; EML, extramedullary leukemia; CNSL, central central nervous system leukemia; SDAC, standard dose Ara-C; MDAC, median dose Ara-C; CR, complete response; OS, overall survival; DFS, disease free survival; WBC, white blood cell count; HR, hazard ratio; CI, confidence interval; ACAs, additional chromosomal abnormalities; CIR, cumulative incidence of relapse; WT, wild type; APP, amyloid precursor protein.

\section{Introduction}

The AML1-ETO (RUNX1-RUNX1T1; AE) fusion protein generated from translocation at chromosome 8 and 21 represents one of the most common cytogenetic abnormalities in acute myeloid leukemia (AML), accounting for $5 \sim 10 \%$ of all cases [1-2]. Although the AML1-ETO-positive AML (AE-AML) is considered a subtype with favorable prognosis, studies have shown that the AE-AML is highly heterogenous, shown as up to $6-31 \%$ of AE-AML patients carrying a c-KIT mutation and 15-26.7\% presenting with extramedullary 
leukemia (EML) [1, 3-4], also the long term survival ranging from 28 to $70 \%$ [4-6]. While most research report both c-KIT mutations and EML are associated with higher risk of relapse and worse survival in AE-AML [3-4, 7], some studies, especially in children, show neither seems to impact the disease outcome [8-9], which in turn further supports the heterogeneity of this subtype leukemia. Taking together, it suggests AML1-ETO fusion protein might behave heterogenously in different subgroups, might be age-dependent. However, the different clinical significance of c-KIT mutation and EML between pediatric and adult patients with AE-AML remains unsure. Further study is needed to reveal the different phenotype of AE-AML in pediatric and adult patients, which is good for better understanding of the heterogeneity of AML1-ETO fusion protein.

In this multi-center retrospective study, we analyzed the demographic features of pediatric vs. adult patients in AE-AML, and demonstrated the different phenotype of c-KIT mutation and EML with age-dependence.

\section{Patients and methods}

\section{Patient characteristics}

A total of 173 newly diagnosed AE-AML patients from Nanfang Hospital, Southern Medical University, Sun Yat-sen Memorial Hospital Sun Yat-sen University, Zhujiang Hospital, Southern Medical University, The First Affiliated Hospital of Guangzhou Medical University and Guangzhou Women and Children's Medical Center in Guangzhou, China from January 2006 to December 2017 were retrospectively reviewed. Seventy five were children ([?]14-year old) and 98 were adults (>14 years old). Patients' clinical features were shown in Table 1. All tissue samples and clinical data were obtained upon the approval of Medicine Institutional Review Boards of related centers. Written informed consent was obtained from all patients and/or guardians.

\section{Diagnosis and classification}

The morphology of bone marrow and peripheral blood was reviewed following Wright-Giemsa staining and classified according to the French-American-British (FAB) classification. Immunophenotype was analyzed by flow cytometry. The cytogenetics was established by chromosome banding combined with fluorescence in situ hybridization (FISH) [7]. The diagnosis of AE-AML was made based on WHO 2016 classification [10]. EML included extramedullary infiltration (EMI) and central central nervous system leukemia (CNSL), which was defined according to the previous report by Støve HK et al [11].

\section{Gene mutation analysis.}

Gene sequencing of Fms related tyrosine kinase 3 (FLT3)- internal tandem duplication (ITD) , FLT3tyrosine kinase domain (TKD), c-KIT (exons 8 and 17) and NPM1 was performed by polymerase chain reaction $(\mathrm{PCR})$ and direct Sanger sequencing according to our previous study $[7,12]$.

\section{Therapy}

Anthracyclines, including daunorubicin (DNR), idarubicin (IDA), mitoxantrone (Mito), pirarubicin (THP) or homoharringtonine (HHT), combined with cytarabine (Ara-C), FLAG (fludarabine, cytarabine, granulocyte colony stimulating factor) combined with idarubicin and DAE (DA combined with etoposide) were main induction therapies. Consolidation regimens included standard dose Ara-C (SDAC)-based and median dose Ara-C (MDAC)-based regimens, as previously reported [4, 7]. ICE (idarubicin, cytarabine, etoposide), CAG (aclarubicin, cytarabine, granulocyte-colony stimulating factor), FLAG and CLARA (cladribine and cytarabine) were used as salvage therapies. Allo-transplant was mainly performed in patients carrying c-KIT mutations and in those who had not achieved complete response (CR) after two cycles of induction therapies.

\section{Survival and statistical analysis}

The overall survival (OS) was calculated from the day of diagnosis to the day of death or the last follow-up, and the disease free survival (DFS) from the day of diagnosis to the day of relapse or the last follow-up. CR was defined as bone marrow blasts less than $5 \%$ irrespective of peripheral blood cell recovery. 
Statistical analyses were performed using SPSS 20.0. Not normally distributed data were described by the median. A comparison of distribution between two groups of data was performed by a Mann-Whitney nonparametric U test, and correlations between two variables were performed by Spearman rank correlation analysis. The Kaplan-Meier method was used to estimate median survival and survival probabilities, and associations with covariates were tested by the log-rank test. Multivariate analysis was performed to identify independent prognostic factors adopting Cox regression model. $\mathrm{P}<0.05$ was considered to indicate a statistically significant difference.

\section{Results}

\section{Clinical characteristics}

AML-M2 was the most common phenotype, which occurred in $82.7 \%(62 / 75)$ of children and $92.9 \%(91 / 98)$ of adults $(\mathrm{P}=0.005)$. Thirty nine patients $(22.5 \%)$ presented with various forms of EML, including central nervous system leukemia (CNSL) $(n=12)$, sarcoma within or beside the spine $(n=17)$, and others $(n=10)$. Compared to adult group, the pediatric cohort had a much lower incidence of EML (children 10/75, 13.3\% vs. adults $29 / 98,29.6 \%, \mathrm{P}=0.011)$. Of the 39 patients with $\mathrm{EML}$, there were 1 child $(1 / 10,10.0 \%)$ vs. 14 adults $(14 / 29,48.3 \%, \mathrm{P}=0.032)$ presenting with sarcoma within or beside the spine (Table 2). C-KIT mutations were also less frequently observed in children $(12 / 68,17.6 \%)$ than in adults $(30 / 74,40.5 \%, \mathrm{P}=0.003)$. Otherwise, there were no statistical differences with regards to gender, peripheral white blood cell count (WBC), immunphenotype of CD34, CD13, CD33, CD117, HLA-DR, CD19 and CD56 positive expression, cytogenetic abnormality, and gene mutations including FLT3-ITD and NPM1 between the two age groups (Table 1).

\section{Treatment regimens and treatment response}

One hundred and seventy out of 173 patients, including 75 children and 95 adults, received one to two cycles of induction therapies: 103 on anthracyclines combined with cytarabine (AA), 54 on either FLAG+IDA or DAE, and 13 on other regimens. Detail was documented in table 1. AA was the main induction regimen in adults, while intensive regimen FLAG+IDA or DAE was mainly used in children (adults vs. children: AA, $89 / 95,93.7 \%$ vs. $14 / 75,18.7 \%$; FLAG+IDA/DAE, 0 vs. $54 / 75,72.0 \%, \mathrm{P}<0.001$ ). After two cycles of induction therapies, cumulatively 155/170 $(91.1 \%)$ patients achieved CR. CR was much higher in children (children $73 / 75,97.3 \%$ vs. adults $82 / 95,86.3 \%, \mathrm{P}=0.012$ ). For those who received AA regimen, there was no statistical difference in CR rate between the two age groups (children 13/14, 92.9\% vs. adults 78/89, 87.6\%, $\mathrm{P}=0.572)$.

Univariate analysis revealed that age (hazard ratio [HR] 5.787, 95\% confidence interval [CI] 1.263-26.504, $\mathrm{P}=0.024$ ) and FLT3-ITD mutation (HR 6.100, 95\% CI 1.323-28.129, $\mathrm{P}=0.020$ ), but not gender, EML, peripheral WBC count, immunphenotype, additional chromosomal abnormalities (ACAs) or c-KIT mutations, were the adverse factors for CR. Multivariate analysis showed that the FLT3-ITD mutation (HR 23.240, 95\% CI 1.202-449.293, $\mathrm{P}=0.037$ ) was the only independent poor prognostic factor. However, when induction therapies were taken into account, none of the above were identified as unfavorable per multivariate analysis $(\mathrm{P}>0.05)$.

Cumulatively, a total of 160 out of $170(94.1 \%)$ patients achieved CR. The CR in children occurred in $74 / 75(98.7 \%)$ patients and that in adults was much lower in $86 / 95(90.5 \%)$ patients $(\mathrm{P}=0.025)$. All CR patients then received a median of $4(1-11)$ cycles of consolidation therapies. Pediatric patients were mainly treated with MDAC-based regimen (55/74, 74.3\%), while SDAC-based (47/86, 54.7\%) and MDAC-based regimens $(39 / 86,45.3 \%)$ were given to adult patients $(\mathrm{P}<0.001)$. Patients who required salvage therapy or allo-transplant were comparable between the two age groups (Table 1).

With a median follow-up of 20 (2-96) months, 112 out of 170 patients survived and 58 died: 40 from disease relapse, 10 from lack of response, and 8 due to treatment-related mortality. Of the 160 patients who achieved CR, 55 relapsed. For the entire study cohort, the cumulative incidence of relapse (CIR) was $28.3 \% \pm 3.9 \%$, the DFS $65.4 \% \pm 3.9 \%$ and OS $69.9 \% \pm 3.8 \%$. The 2 -year CIR, DFS and OS were $32.8 \% \pm 4.1 \%, 60.5 \% \pm 4.1 \%$ 
and $65.5 \% \pm 4.1 \%$, respectively.

\section{Relapse}

The CIR in pediatric patients was significantly lower than that in adult patients $(21.8 \% \pm 5.4 \%$ vs. $34.1 \% \pm 5.5 \%, \mathrm{P}=0.037$, figure $1 \mathrm{~A} 1)$. The difference in CIR was also seen at 2 year interim $(30.8 \% \pm 6.4 \%$ in children vs. $43.1 \% \pm 6.1 \%$ in adults, $\mathrm{P}=0.046$, supplemental figure $1 \mathrm{~A})$. Consistent with previous report [13], MDAC-based consolidation greatly lowered disease relapse compared to SDAC-based regimen $(\mathrm{P}<0.001$, supplemental figure 2). To investigate the impact of age on CIR, we analyzed the CIR in patients receiving MDAC- vs. SDAC-based regimens respectively. When consolidation regimens were taken into consideration, there was no statistical difference in CIR between the two age cohorts (MDAC-based regimen: children $11.9 \% \pm 5.0 \%$ vs. adults $16.3 \% \pm 6.7 \%, \mathrm{P}=0.467$; SDAC-based regimen: children $45.8 \% \pm 12.0 \%, \mathrm{P}=0.517$ vs. adults $47.5 \% \pm 7.6 \%$, figure $1 \mathrm{~A} 2$ and $\mathrm{A} 3)$.

Multivariate analysis indicated that both EML (with vs. without, HR 2.20, 95\% CI 1.11-4.35, $\mathrm{P}=0.024$ ) and consolidation regimens (MDAC-based vs. SDAC-based, HR 0.20, 95\% CI 0.10-0.39, P<0.001) were independent risk factors for disease relapse. Patients harboring c-KIT mutations (mutant vs. wild type [WT], HR 1.80, 95\% CI 0.92-3.50, $\mathrm{P}=0.088$ ) also showed a trend toward higher risk of recurrence, although this was not statistically significant. Other variables, including gender (male vs. female), age ([?]14 vs. ¡14 years),

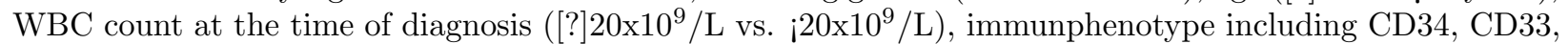
CD13, CD117, HLA-DR, CD19 and CD56 (positive vs. negative), additional chromosome abnormalities (with vs. without) and FLT3-ITD status (mutant vs. WT) did not affect disease recurrence. Detail was shown in table 3.

To examine whether EML and c-KIT mutations behaved similarly on relapse in terms of patients' age, we performed subgroup analysis which confirmed EML as the risk factor for relapse only in adult patients (with $54.6 \%+-10.8 \%$ vs. without $26.0 \%+-6.1 \%, \mathrm{P}=0.002$, figure $2 \mathrm{~A} 2$ ), but not in pediatric patients (with $38.3 \%+-$ $18.0 \%$ vs. without $19.3 \%+-5.5 \%, \mathrm{P}=0.082$, figure $2 \mathrm{~A} 3$ ). Similarly, c-KIT mutations were associated with higher risk of relapse only in adults (adults, mutant $52.4 \%+-10.2 \%$ vs. WT $22.8 \%+-7.3 \%, \mathrm{P}=0.005$, figure $3 \mathrm{~A} 2$; children, mutant $20.0 \%+-17.9 \%$ vs. WT $23.6 \%+-6.3 \%, \mathrm{P}=0.372$, figure $3 \mathrm{~A} 3$ ). The adverse effects of EML and c-KIT mutations on disease recurrence in adults were not improved by choice of consolidation regimens (supplemental figure 3 and 4 ).

\section{Disease free survival}

The DFS was significantly higher in pediatric group than in adult group (children $74.3 \%+-5.6 \%$ vs. adults $58.2 \%+-5.4 \%, \mathrm{P}=0.021$, figure $1 \mathrm{~B} 1$ ). The DFS benefit in children was also seen at 2 year interim (children $66.9 \%+-6.1 \%$ vs. adults $55.3 \%+-5.5 \%, \mathrm{P}=0.014$, supplemental figure 1B). However, when consolidation regimens were taken into consideration, the DFS was comparable between the two age groups (MDACbased regimen: children $86.2 \%+-5.3 \%, \mathrm{P}=0.959$ vs. adults $83.7 \%+-6.7 \%$, figure 1B2; SDAC-based regimen: children $50.3 \%+-11.8 \%$ vs. adults $50.0 \%+-7.7 \%, \mathrm{P}=0.581$, figure $1 \mathrm{~B} 3$ ).

Based on multivariate analysis, both EML (with vs. without, HR 1.88, 95\% CI 0.97-3.64, P=0.048) and consolidation regimens (MDAC-based vs. SDAC-based, HR 0.22,95\% CI 0.12-0.42, $\mathrm{P} j 0.001$ ) were independent risk factors for DFS. Being positive in c-KIT mutations (mutant vs.WT, HR 1.80, 95\% CI 0.95-3.40, P=0.095) was also associated with a trend toward lower DFS. Variables, including gender (male vs. female), age ([?]14

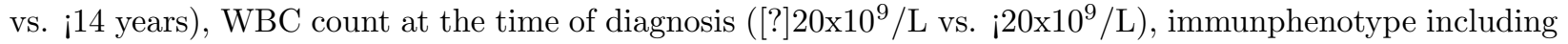
CD34, CD33, CD13, CD117, HLA-DR, CD19 and CD56 (positive vs. negative), additional chromosome abnormalities (with vs. without) and FLT3-ITD status (mutant vs. WT) did not affect DFS. Detail was shown in table 3.

Multivariate analysis revealed that the adverse effects of EML and c-KIT mutations on DFS were only seen in adults (EML: with $38.7 \%+-9.7 \%$ vs. without $66.6 \%+-6.2 \%$, $\mathrm{P}=0.003$, figure $2 \mathrm{~B} 2$; c-KIT mutations: mutant $44.5 \%+-9.7 \%$ vs. WT $68.5 \%+-7.8 \%, \mathrm{P}=0.015$, figure 3B2), but not in children (EML: with $61.7 \%+-18.0 \%$ vs. without $76.2 \%+-5.8 \%, \mathrm{P}=0.205$, figure B3; c-KIT mutations: mutant $68.6 \%+-18.6 \%$ vs. WT $73.3 \%+-$ 
$6.4 \%, \mathrm{P}=0.602$, figure 3B3). The inferior outcome in adult cohort with EML and c-KIT mutations was not improved by consolidation treatment (supplemental figure 5 and 6 ).

\section{Overall survival}

Pediatric patients enjoyed better OS than adults (adults $59.5 \%+-5.4 \%$ vs. children $83.2 \%+-4.9 \%, \mathrm{P}=0.001$, figure $1 \mathrm{C} 1$ ). The survival benefit in children was also observed at 2 year interim (adults $56.5 \%+-5.5 \%$ vs. children $77.0 \%+-5.7 \%, \mathrm{P}=0.005$, supplemental figure $1 \mathrm{C}$ ). There was no difference in OS between the two groups when consolidation regimens were taken into consideration (MDAC-based regimen: children $88.2 \%+-$ $5.5 \%$ vs. adults $84.0 \%+-7.3 \%, \mathrm{P}=0.575$, figure $1 \mathrm{C} 2$; SDAC-based regimen: children $60.0 \%+-12.6 \%$ vs. adults $45.0 \%+-7.9 \%, \mathrm{P}=0.277$, figure $1 \mathrm{C} 3)$.

By multivariate analysis, both EML (with vs. without, HR 2.14, 95\% CI 1.13-4.06, $\mathrm{P}=0.020$ ) and consolidation regimens (MDAC-based vs. SDAC-based, HR 0.18, 95\% CI 0.09-0.36, $\mathrm{P}<0.001$ ) were independent risk factors for OS. C-KIT mutations (mutant vs.WT, HR 1.80, 95\% CI 0.96-3.35, $\mathrm{P}=0.065$ ) tended to be a poor factor for OS. Variables, including gender (male vs. female), age ([?]14 vs. ¡14 years), WBC count at the time of diagnosis ([?]20x109/L vs. <20x109/L), immunphenotype including CD34, CD33, CD13, CD117, HLA-DR, CD19 and CD56 (positive vs. negative), additional chromosome abnormalities (with vs. without) and FLT3-ITD status (mutant vs. WT) did not affect the OS. Detail was shown in table 3.

Subgroup analysis revealed that adverse effects of EML (adults: with $38.7 \%+-9.7 \%$ vs. without $68.4 \%+-$ $6.1 \%, \mathrm{P}=0.001$, figure $2 \mathrm{C} 2$; children: with $68.6 \%+-18.6 \%$ vs. without $85.0 \%+-4.9 \%, \mathrm{P}=0.579$, figure $2 \mathrm{C} 3$ ) and c-KIT mutations (adults: mutant $44.5 \%+-9.7 \%$ vs. WT $68.5 \%+-7.8 \%, \mathrm{P}=0.021$, figure $3 \mathrm{C} 2$; children: mutant $85.7 \%+-13.2 \%$ vs. WT $82.5 \%+-5.7 \%, \mathrm{P}=0.537$, figure $3 \mathrm{C} 3$ ) on OS were only observed in adult patients. The negative impact of EML and c-KIT mutations on OS was irrespective of consolidation therapies (supplemental figure 7 and 8).

\section{Discussion}

We retrospectively analyzed the clinical features of pediatric vs. adult patients with AE-AML. Our study revealed that both EML and c-KIT mutations were more frequently seen in adult AE-AML. Accordingly, this group of patients carried a higher risk of relapse, and had a lower rate of DFS and OS compared to the pediatric counterparts.

In line with other's study [13], the AML-M2 was the dominant clinical subtype occurring in $88.2 \%$ of patients in our cohort. It was even higher in up to $92.9 \%$ of adult patients. Apart from AML-M2 phenotypic dominance, high incidence of EML and c-KIT mutations were other two features in AE-AML [1,3-4]. In our study, 22.5\%(39/173) patients presented with EML and 29.6\%(42/142) carried a c-KIT mutation. Subgroup analysis demonstrated that the occurrence of EML ( $29.6 \%$ vs. $13.3 \%, \mathrm{P}=0.008)$ and c-KIT mutations $(40.5 \%$ vs. $17.6 \%, \mathrm{P}=0.003)$ were at least doubled in adult group compared to pediatric cohort.

According to previous studies, both c-KIT mutations [7,11] and EML [7,14-15] were associated with an increased expression of amyloid precursor protein (APP), which adversely impacted the disease outcome in AE-AML. APP is a type I integral membrane protein abundant in neurons [16]. It was reported to be overexpressed in solid tumors, including oral squamous cell carcinoma, pancreatic adenocarcinoma and prostate cancer, promoting cell proliferation and metastasis therefore adversely affecting the prognosis [17-19]. The APP gene is located on 21q21.3. The app mRNA was over-expressed in AML with abnormal chromosomal 21 [20], i.e. t (8;21) AML [21], as compared with other AML subtypes. Jiang et al reported the role of APP in cell migration through the APP/phosphorylated extracellular-signal-regulated kinase (p-ERK)/cMyc/matrix metalloproteinase (MMP)-2 pathway and its involvement in the pathogenesis of EML [14-15]. Consistent with other studies [22-23], the majority of EML in our study presented with CNS infiltration in the form of either sarcoma within or beside the spine $(15 / 39,38.5 \%)$ or CNSL $(15 / 39,38.5 \%)$. The CNS infiltration of EML, in particular the sarcoma within or beside the spine, occurred at a much higher rate in adults than that in children (adults $14 / 29,48.3 \%$ vs. children $1 / 10,10.0 \%, \mathrm{P}=0.032$ ). In addition, in AE-AML the overepression of APP was associated with both a higher incidence of c-KIT mutations and a 
higher level of c-kit expression [7,11]. The critical step of APP proteolysis in Alzheimer's disease [16] which is mainly an elders' illness, and the overexpression of APP in certain solid tumors which also mainly affect the elders made us speculate that the expression of APP might increase with aging. Hence the variance in EML and c-KIT mutations between the two age groups might reflect the underlying difference in APP expression. Furthermore, the present study, further supporting this hypothesis, from the clinical point of view, showed that EML and c-KIT mutation negatively impacted on relapse, DFS and OS, and the poor impact was only found in adult but not pediatric patients by subgroup analysis, which was in agreement with the adverse prognostic effect of APP in AE-AML [7,14-15].

EML has an adverse prognostic effect in adult patients with AE-AML [7,14-15, 24-25]. However, its prognostic effect seems not so important in pediatric AE-AML $[9,26]$, though it might a poor prognostic factor in pediatric patients with AML in the whole cohort [11,27]. In the present study, EML was associated with a higher risk of relapse (HR 2.20, 95\% CI 1.11-4.35, $\mathrm{P}=0.024$ ), and worse DFS (HR 1.88, 95\% CI 0.97-3.64, $\mathrm{P}=0.048$ ) and $\mathrm{OS}$ (HR 2.14, 95\% CI 1.13-4.06, $\mathrm{P}=0.020$ ). However, the negative effects of EML on relapse and survival were only seen in adult patients $(\mathrm{P}<0.05)$ but not in children $(\mathrm{P}>0.05)$. Our results further supported that EML had negative prognostic effect only in adult but not pediatric AE-AML. The consolidation regimens did not improve the poor outcome in adults presenting with EML.

Mutations in c-kit are one of most common genetic abnormalities, especially in AE-AML. While most studies showed the presence of c-KIT mutations adversely impacted the survival in AE-AML [1-3,28], some research also reported that it did not influence the outcome especially in pediatric patients [29-30]. In our study, the presence of c-KIT mutations did not affect the whole cohort in terms of relapse (HR 1.80, 95\% CI 0.92-3.50, $\mathrm{P}=0.088$ ), DFS (HR 1.80, 95\% CI 0.95-3.40, $\mathrm{P}=0.095$ ) and OS (HR 1.80, 95\% CI 0.96-3.35, $\mathrm{P}=0.065$ ). It was, however, an unfavorable factor for relapse and survival in adults $(\mathrm{P}<0.05)$, and the treatment regimens were not able to improve the prognosis in this group of patients. Taken together, EML and c-KIT mutations adversely affected the disease outcome only in adult but not pediatric AE-AML.

When compared to SDAC-based regimens, median or high dose cytarabine-based regimens have improved the survival and lowered the relapse of AE-AML [12,31], and are the consolidation treatment recommended by guidelines [32-34]. Our data further supported the strength of MDAC-based regimen as the consolidation therapy irrespective of patients' age, with the only exception of those with either EML or c-KIT mutations. The high CR in pediatric patients was mainly due to intensive induction regimens, and the CR rates between the two age groups became comparable when patients were given similar induction regimens. Our study suggested that the patients with AE-AML could benefit from intensive induction and consolidation regimens, but such benefit was not seen in high-risk patients. Allogenic stem cell transplantation should be recommended to adult patients with EML and c-KIT mutations [35].

\section{Conclusion}

AML-M2 was the dominant morphology in AE-AML. The incidence of EML and c-KIT mutations were much higher in adult patients and adversely affected the disease outcome in this group of patients, suggesting AML1-ETO fusion gene might behave differently in adults and children. While the intensive induction and consolidation therapy were considered to benefit patients of AE-AML regardless of age, their use have not improved the prognosis of adult patients either presenting with EML or harboring a c-KIT mutation. Studies

are required in the future to explore the underlying pathogenesis of EML and c-KIT mutation in pediatric and adult AE-AML.

\section{Acknowledgments}

This study was funded by the National Natural Science Foundation of China (no. 81870128), the Natural Science Foundation of Guangdong Province, China (no. 2020A1515011514), the Key Platforms and Scientific Research Projects of Guangdong Universities of Guangdong Provincial Department of Education (no. 2018KTSCX034), the Clinical Research Initiative of Southern Medical University (no. LC2016YM006) and the President Funding of Nanfang Hospital (no. 2018Z021). 


\section{Authors contributions}

GP, ZY, YY, JZ, JQ, JZ, CY, DF, XX and DX made substantial contributions to the conception of the study, were involved in the analysis and interpretation of the data. All authors drafted or substantively revised the manuscript, approved the final manuscript for submission and agree to be accountable for all aspects of the research in ensuring that the accuracy or integrity of any part of the work are appropriately investigated and resolved.

\section{Conflicts of Interest}

The authors have no conflicts of interest to declare.

\section{Ethical Statement}

The study protocol conformed to the ethical guidelines of the Declaration of Helsinki. All tissue samples and clinical data were obtained upon the approval of Medicine Institutional Review Boards of related centers. Written informed consent was obtained from all patients and/or guardians. The content of the informed consent included the agreement of patient clinical data being published in any form.

\section{Reference}

1. Park SH, Chi HS, Cho YU, et al. Effects of c-KIT mutations on expression of the RUNX1/RUNX1T1 fusion transcript in t(8;21)positive acute myeloid leukemia patients. Leuk Res. 2013;37: 784-789.

2. Muller AM, Duque J, Shizuru JA, Lubbert M. Complementing mutations in core binding factor leukemias: from mouse models to clinical applications. Oncogene. 2008;27:5759-5773.

3. Krauth MT, Eder C, Alpermann T, et al. High number of additional genetic lesions in acute myeloid leukemia with t(8;21)/RUNX1RUNX1T1: Frequency and impact on clinical outcome. Leukemia. 2014;28:1449-1458.

4. Yu G, Yin $\mathrm{C}, \mathrm{Wu} \mathrm{F}$, et al. Gene mutation profile and risk stratification in AML1ETO-positive acute myeloid leukemia based on nextgeneration sequencing. Oncol Rep. 2019; 42: 2333-2344.

Duployez N, Marceau-Renaut A, et al. Comprehensive mutational profiling of core binding factor acute myeloid leukemia. Blood. 2016;127: 2451-2459. 6. Qin YZ, Zhu HH, Jiang Q, et al. Prevalence and prognostic significance of c-KIT mutations in core binding factor acute myeloid leukemia: A comprehensive large-scale study from a single Chinese center. Leuk Res. 2014;38:1435-1440.

7. Yu G, Yin C, Jiang L, et al. Amyloid precursor protein has clinical and prognostic significance in AML1ETOpositive acute myeloid leukemia. Oncol Lett. 2018;15: 917-925.

8. Klein K, Kaspers G, Harrison CJ, et al. Clinical Impact of Additional Cytogenetic Aberrations, cKIT and RAS Mutations, and Treatment Elements in Pediatric t(8;21)-AML: Results From an International Retrospective Study by the International Berlin-Frankfurt-Munster Study Group. J Clin Oncol. 2015;33:42474258 .

9. Abdel RH, Farrag SA, El-Attar IA. AML1/ETO Fusion Gene in de novo Pediatric Acute Myeloid Leukemia: Clinical Significance and Prognostic Implications. J Egypt Natl Canc Inst. 2007;19:39-47.

10. Arber DA, Orazi A, Hasserjian R, et al. The 2016 revision to the world health organization classification of myeloid neoplasms and acute leukemia. Blood. 2016;127: 2391-2405.

Stove HK, Sandahl JD, Abrahamsson J, et al. Extramedullary leukemia in children with acute myeloid leukemia: A population-based cohort study from the Nordic Society of Pediatric Hematology and Oncology (NOPHO). Pediatr Blood Cancer. 2017;64(12). doi: 10.1002/pbc.26520.

12. Yu G, Yin C, Jiang L, et al. Amyloid precursor protein cooperates with C-KIT mutation/overexpression to regulate cell apoptosis in AML1-ETO-positive leukemia via PI3K/AKT signaling pathway. Oncol Rep. 2016;36:1626-1632. 
13. Yin JA, O'Brien MA, Hills RK, et al. Minimal residual disease monitoring by quantitative RT-PCR in core binding factor AML allows risk stratification and predicts relapse: results of the United Kingdom MRC AML-15 trial. Blood. 2012;120:2826-2835.

14. Jiang $\mathrm{L}, \mathrm{Yu} \mathrm{G}$, Meng $\mathrm{W}$, et al. Overexpression of amyloid precursor protein in acute myeloid leukemia enhances extramedullary infiltration by MMP-2. Tumour Biol. 2013; 34: 629-636.

15. Jiang L, Meng W, Yu GP, et al. MicroRNA144 targets APP to regulate AML1/ETO+ leukemia cell migration via the pERK/cMyc/MMP2 pathway. Oncol lett. 2019; 18: 2034-2042.

16. Zhang MY, Zheng CY, Zou MM, et al. Lamotrigine attenuates deficits in synaptic plasticity and accumulation of amyloid plaques in APP/PS1 transgenic mice. Neurobiol Aging. 2014;35:2713-2725.

17. Hansel DE, Rahman A, Wehner S, et al. Increased expression and processing of the Alzheimer amyloid precursor protein in pancreatic cancer may influence cellular proliferation. Cancer Res. 2003;63:7032-7037.

18. Ko SY, Lin SC, Chang KW, et al. Increased expression of amyloid precursor protein in oral squamous cell carcinoma. Int J Cancer. 2004;111:727-732.

19. Takayama K, Tsutsumi S, Suzuki T, et al. Amyloid precursor protein is a primary androgen target gene that promotes prostate cancer growth. Cancer Res. 2009;69:137-142.

20. Baldus CD, Liyanarachchi S, Mrozek K, et al. Acute myeloid leukemia with complex karyotypes and abnormal chromosome 21: Amplification discloses overexpression of APP, ETS2, and ERG genes. Proc Natl Acad Sci USA. 2004;101:3915-3920.

21. Wang W, Meng FY, Huang ZF, et al. Expression and role of amyloid precrusor protein gene in acute myeloid leukemia. Chin J Hematol. 2010;31:309-14 (In Chinese).

22. Rubnitz JE, Raimondi SC, Hallbert AR, et al. Characteristics and outcome of t(8;21)-positive childhood acute myeloid leukemia: A single institution's experience. Leukemia. 2002;16:2072-2077.

23. Lan TY, Lin DT, Tien HF, et al. Prognostic factors of treatment outcomes in patients with granulocytic sarcoma. Acta Haematol. 2009;122:238-246.

24. Byrd JC,Weiss RB, Arthur DC, at al.Extramedullary leukemia adversely affects hematologic complele remission rate and overall survival in patients with $\mathrm{t}(8 ; 2$ 1)(q22;q22):results from Cancer and Leakemia Group B8461.J Clin Oncol. 1997; 15: 466-475.

25. Lai YY, Qiu JY, Jiang B, et al. Characteristics and prognostic factors of acute myeloid leukemia with t( 8; 21) ( q22; q22). Journal of Experimental Hematology. 2005; 13( 5) : 733- 740.

26. Felice MS, Zubizarreta PA, Alfaro EM, et al. Good outcome of children with acute myeloid leukemia and $\mathrm{t}(8 ; 21)$ (q22; $\mathrm{q} 22)$, even when associated with granulocytic sarcoma: a report from a single institution in Argentina. Cancer. 2000; 88(8):1939-1944.

27. Kobayashi R, Tawa A, Hanada R, et al. Extramedullary infiltration at diagnosis and prognosis in children with acute myelogenous leukemia. Pediatr Blood Cancer. 2007;48(4):393-398.

28. Voigt AP, Brodersen LE, Alonzo TA, et al. Phenotype in combination with genotype improves outcome prediction in acute myeloid leukemia: a report from Children's Oncology Group protocol AAML0531. Haematologica. 2017;102:2058-2068.

29. Pollard JA, Alonzo TA, Gerbing RB, et al. Prevalence and prognostic significance of KIT mutations in pediatric patients with core binding factor AML enrolled on serial pediatric cooperative trials for de novo AML. Blood. 2010;115:2372-2379.

30. Shih LY, Liang DC, Huang CF, et al. Cooperating mutations of receptor tyrosine kinases and Ras genes in childhood core-binding factor acute myeloid leukemia and a comparative analysis on paried diagnosis and relapse samples. Leukemia. 2008;22:303-307. 
31. Lowenberg B, Pabst T, Vellenga E, et al. Cytarabine dose for acute myeloid leukemia. N Engl J Med. 2011;364:1027-1036.

32. Acute myeloid leukemia, Version 3.2020, 12/23/19 (c) 2019 National Comprehensive Cancer Network(r) $(\mathrm{NCCN}(\mathrm{r}))$. http://guide.medlive.cn.

33. Leukemia \& Lymphoma Group, Chinese Society of Hematology, Chinese Medical Association. Chinese guildlines for diagnosis and treatment of adult acute lyeloid leukemia (not APL) (2017). Chin J Hematol. 2017;38:177-184.

34. Dohner H,Estey E,Grimwade D, et al. Diagnosis and management of AML in adults: 2017 ELN recommendations from an international expert panel. Blood. 2017;129:424-447.

35. Zhu HH, Zhang XH, Qin YZ, et al. MRD-directed risk stratification treatment may improve outcomes of $\mathrm{t}(8 ; 21)$ AML in the first complete remission: results from the AML05 multicenter trial. Blood. 2013;121:40564062 .

Figure 1. CIR, DFS and OS in the pediatric and adult patients.(1) CIR: children vs. adults: $21.8 \%+-5.4 \%$ vs. $34.1 \%+-5.5 \%, \mathrm{P}=0.037$ (A1); MDAC-based regimen: children $11.9 \%+-5.0 \%$ vs. adults $16.3 \%+-6.7 \%, \mathrm{P}=0.467$ (A2); SDAC-based regimen: children $45.8 \%+-12.0 \%$ vs. adults $47.5 \%+-7.6 \%$, $\mathrm{P}=0.517$ (A3). (2) DFS: children vs. adults: $74.3 \%+-5.6 \%$ vs. $58.2 \%+-5.4 \%, \mathrm{P}=0.021$ (B1); MDACbased regimen: children $86.2 \%+-5.3 \%$ vs. adults $83.7 \%+-6.7 \%, \mathrm{P}=0.959$ (B2); SDAC-based regimen: children $50.3 \%+-11.8 \%$ vs. adults $50.0 \%+-7.7 \%, \mathrm{P}=0.581$ (B3). (3) OS: children vs. adults: $83.2 \%+-4.9 \%$ vs. $59.5 \%+-5.4 \%, \mathrm{P}=0.001$ (C1); MDAC-based regimen: children $9.1 \%+-4.7 \%$ vs. adults $87.0 \%+-6.1 \%$, $\mathrm{P}=0.701$ (C2); SDAC-based regimen: children $63.4 \%+-12.0 \%$ vs. adults $50.0 \%+-7.7 \%, \mathrm{P}=0.157$ (C3).

Supplemental figure 1. CIR, DFS and OS in pediatric and adult patients at two year interim. (1) 2-year CIR: children $30.8 \%+-6.4 \%$ vs. adults $43.1 \%+-6.1 \%, \mathrm{P}=0.046$ (A); (2) 2-year DFS: children $66.9 \%+-6.1 \%$ vs. adults $55.3 \%+-5.5 \%, \mathrm{P}=0.014$ (B); (3) 2-year OS: children $77.0 \%+-5.7 \%$ vs. adults $56.5 \%+-5.5 \%, \mathrm{P}=0.005(\mathrm{C})$.

Supplemental figure 2. The effect of consolidation regimens on CIR, DFS and OS. (1) CIR: MDAC-based $13.6 \%+-4.0 \%$ vs. SDAC-based $47.2 \%+-6.5 \%, \mathrm{P}<0.001$ (A); (2) DFS: MDAC-based $85.3 \%+-$ $4.1 \%$ vs. SDAC-based 49.6\%+-6.5\%, $\mathrm{P}<0.001$ (B); (3) OS: MDAC-based 90.6\%+-3.4\% vs. SDAC-based $53.6 \%+-6.5 \%, \mathrm{P}<0.001(\mathrm{C})$.

Figure 2. The effect of EML on CIR, DFS and OS. (1) CIR: Total, with $49.9 \%+-9.4 \%$ vs. without $22.6 \%+-4.1 \%, \mathrm{P}=0.000$ (A1); Adults, with $54.6 \%+-10.8 \%$ vs. without $26.0 \%+-6.1 \%, \mathrm{P}=0.002$ (A2); Children, with $38.3 \%+-18.0 \%$ vs. without $19.3 \%+-5.5 \%, \mathrm{P}=0.082$ (A3). (2) DFS: Total, with $44.6 \%+-8.7 \%$ vs. without $71.3 \%+-4.3 \%, \mathrm{P}=0.000$ (B1); Adults, with $38.7 \%+-9.7 \%$ vs. without $66.6 \%+-6.2 \%, \mathrm{P}=0.003$ (B2); Children, with $61.7 \%+-18.0 \%$ vs. without $76.2 \%+-5.8 \%, \mathrm{P}=0.205$ (B3). (3) OS: Total, with $45.9 \%+-8.9 \%$ vs. without $76.6 \%+-4.0 \%, \mathrm{P}=0.000$ (C1); Adults, with $38.7 \%+-9.7 \%$ vs. without, $68.4 \%+-6.1 \%, \mathrm{P}=0.001$ (C2); Children, with $68.6 \%+-18.6 \%$ vs. without $85.0 \%+-4.9 \%, \mathrm{P}=0.579$ (C3).

Figure 3. The effect of c-KIT mutation on CIR, DFS and OS. (1) CIR: Total, mutation $42.3 \%+8.9 \%$ vs. WT $23.5 \%+4.8 \%, \mathrm{P}=0.009$ (A1); Adults, mutation $52.4 \%+-10.2 \%$ vs. WT $22.8 \%+-7.3 \%, \mathrm{P}=0.005$ (A2); Children, mutation $20.0 \%+-17.9 \%$ vs. WT $23.6 \%+-6.3 \%, \mathrm{P}=0.372$ (A3). (2) DFS: Total, mutation $52.1 \%+8.7 \%$ vs. WT $71.1 \%+5.0 \%, \mathrm{P}=0.014$ (B1); Adults, mutation $44.5 \%+-9.7 \%$ vs. WT $68.5 \%+-7.8 \%$, $\mathrm{P}=0.015$ (B2); Children, mutation $68.6 \%+-18.6 \%$ vs. WT $73.3 \%+-6.4 \%, \mathrm{P}=0.602$ (B3). (3) OS: Total, $55.6 \%+8.5 \%$ vs. WT $76.4 \%+4.7 \%, \mathrm{P}=0.008$ (C1); Adults, mutation $44.5 \%+-9.7 \%$ vs. WT $68.5 \%+-7.8 \%$, $\mathrm{P}=0.021$ (C2); Children, mutation $85.7 \%+-13.2 \%$ vs. WT $82.5 \%+-5.7 \%, \mathrm{P}=0.537$ (C3).

Supplemental figure 3. The effect of EML on CIR in children and adults treated with MDACor SDAC-based regimens. (1) Adults with MDAC-based regimens, with $38.6 \%+-15.3 \%$ vs. without $11.2 \%+-7.5 \%, \mathrm{P}=0.028$ (A1); (2) Adults with SDAC-based regimens, with $70.0 \%+-14.0 \%$ vs. without $39.8 \%+-8.7 \%, \mathrm{P}=0.003$ (A2); (3) Children with MDAC-based regimens, with 0 vs. without $12.6 \%+-5.3 \%$, 
$\mathrm{P}=0.529$ (B1); (4) Children with SDAC-based regimens, with $60.0 \%+-21.9 \%$ vs. without $40.4 \%+-14.1 \%$, $\mathrm{P}=0.377(\mathrm{~B} 2)$.

Supplemental figure 4. The effect of c-KIT mutations on CIR in children and adults treated with MDAC- or SDAC-based regimens. (1) Adults with MDAC-based regimens: mutation 46.7\%+$17.3 \%$ vs. WT $20.0 \%+-17.9 \%, \mathrm{P}=0.003$ (A1); (2) Adults with SDAC-based regimens: mutation $63.3 \%+-$ $12.5 \%$ vs. WT $44.1 \%+-12.2 \%, \mathrm{P}=0.263$ (A2); (3) Children with MDAC-based regimens: mutation 0 vs. WT $14.8 \%+-6.1 \%, \mathrm{P}=0.264$ (B1); (4) Children with SDAC-based regimens: mutation $100 \%$ vs. WT $49.2 \%+-$ $14.4 \% \mathrm{P}=0.991(\mathrm{~B} 2)$.

Supplemental figure 5. The effect of EML on DFS in children and adults treated with MDACor SDAC-based regimens. (1) Adult with MDAC-based regimens: with $61.4 \%+-15.3 \%$ vs. without $88.8 \%+-7.5 \%, \mathrm{P}=0.028$ (A1); (2) Adults with SDAC-based regimens: with $30.0 \%+-14.0 \%$ vs. without $56.9 \%+-8.8 \%, \mathrm{P}=0.004$ (A2); (3) Children with MDAC regimens: with $100 \%$ vs. without $85.4 \%+-5.6 \%$, $\mathrm{P}=0.469$ (B1); (4) Children with SDAC-based regimens: with $40 . \%+-21.9 \%$ vs. without $54.2 \%+-13.8 \%$, $\mathrm{P}=0.483(\mathrm{~B} 2)$.

Supplemental figure 6. The effect of c-KIT mutations on DFS in children and adults treated with MDAC- or SDAC-based regimens. (1) Adults with MDAC-based regimens: mutation 66.7\%+$15.7 \%$ vs. WT $100 \%, \mathrm{P}=0.003$ (A1); (2) Adults with SDAC-based regimens: mutation $36.7 \%+-12.5 \%$ vs. WT 49.7\%+-12.3\%, $\mathrm{P}=0.343$ (A2); (3) Children with MDAC-based regimens: mutation $100 \%$ vs. WT $82.8 \%+-6.4 \%, \mathrm{P}=0.208$ (B1); (4) Children with SDAC-based regimens: mutation 0 vs. WT $50.8 \%+-14.4 \%$, $\mathrm{P}=0.413(\mathrm{~B} 2)$.

Supplemental figure 7. The effect of EML on OS in children and adults treated with MDAC- or SDAC-based regimens. (1) Adults with MDAC-based regimens: with $61.4 \%+-15.3 \%$ vs. without $93.8 \%+-$ $6.1 \%, \mathrm{P}=0.004$ (A1); (2) Adults with SDAC-based regimens: with $30.0 \%+-14.0 \%$ vs. without $56.9 \%+-8.8 \%$, $\mathrm{P}=0.003$ (A2); (3) Children with MDAC-based regimens: with $100 \%$ vs. without $85.4 \%+-5.0 \%, \mathrm{P}=0.589$ (B1). (4) Children with SDAC-based regimens: with $50.0 \%+-25.0 \%$ vs. without $67.5 \%+-13.4 \%, \mathrm{P}=0.863$ (B2).

Supplemental figure 8. The effect of c-KIT mutations on OS in children and adults treated with MDAC- or SDAC-based regimens. (1) Adults with MDAC-based regimens: mutation $66.7 \%+-$ $15.7 \%$ vs. WT $100 \%, \mathrm{P}=0.001$ (A1); (2) Adults with SDAC-based regimens: mutation $36.7 \%+-12.5 \%$ vs. WT $49.7 \%+-12.3 \%, \mathrm{P}=0.449$ (A2); (3) Children with MDAC-based regimens: mutation $100 \%$ vs. WT $87.8 \%+-5.8 \%, \mathrm{P}=0.353$ (B1). (4) Children with SDAC-based regimens: mutation $50.0 \%+-35.4 \%$ vs. WT $62.3 \%+-15.2 \%, \mathrm{P}=0.717(\mathrm{~B} 2)$. 

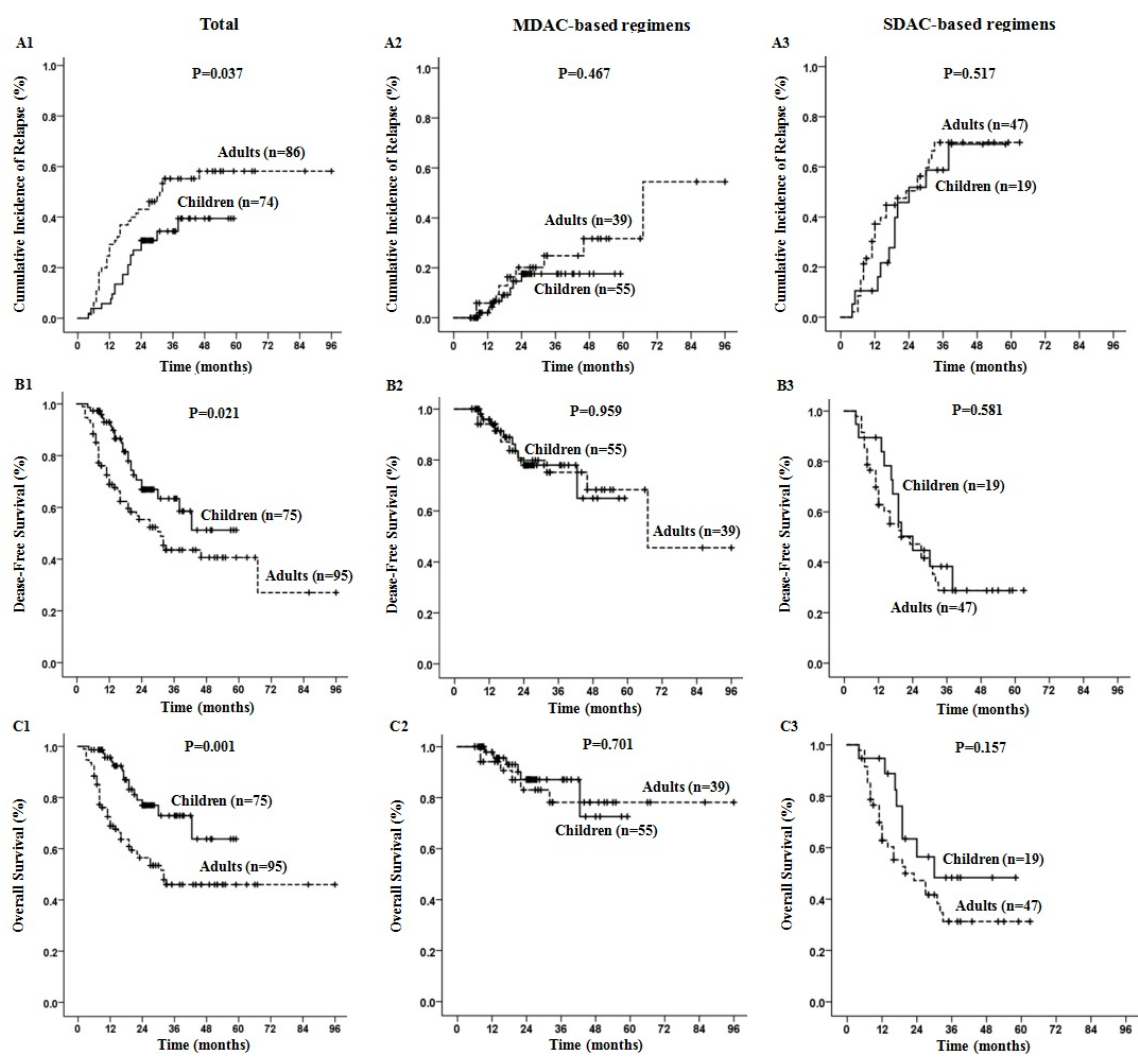

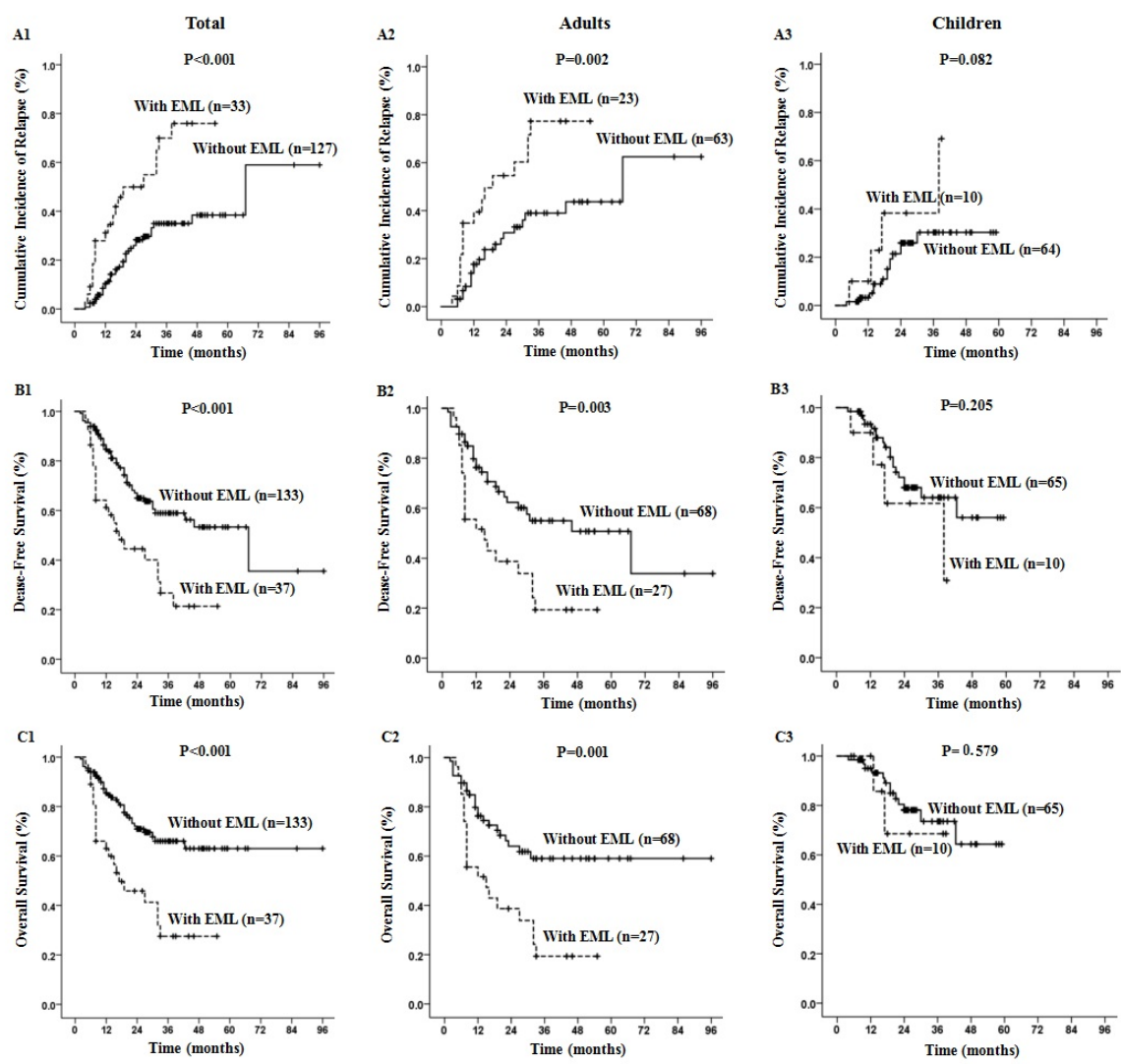

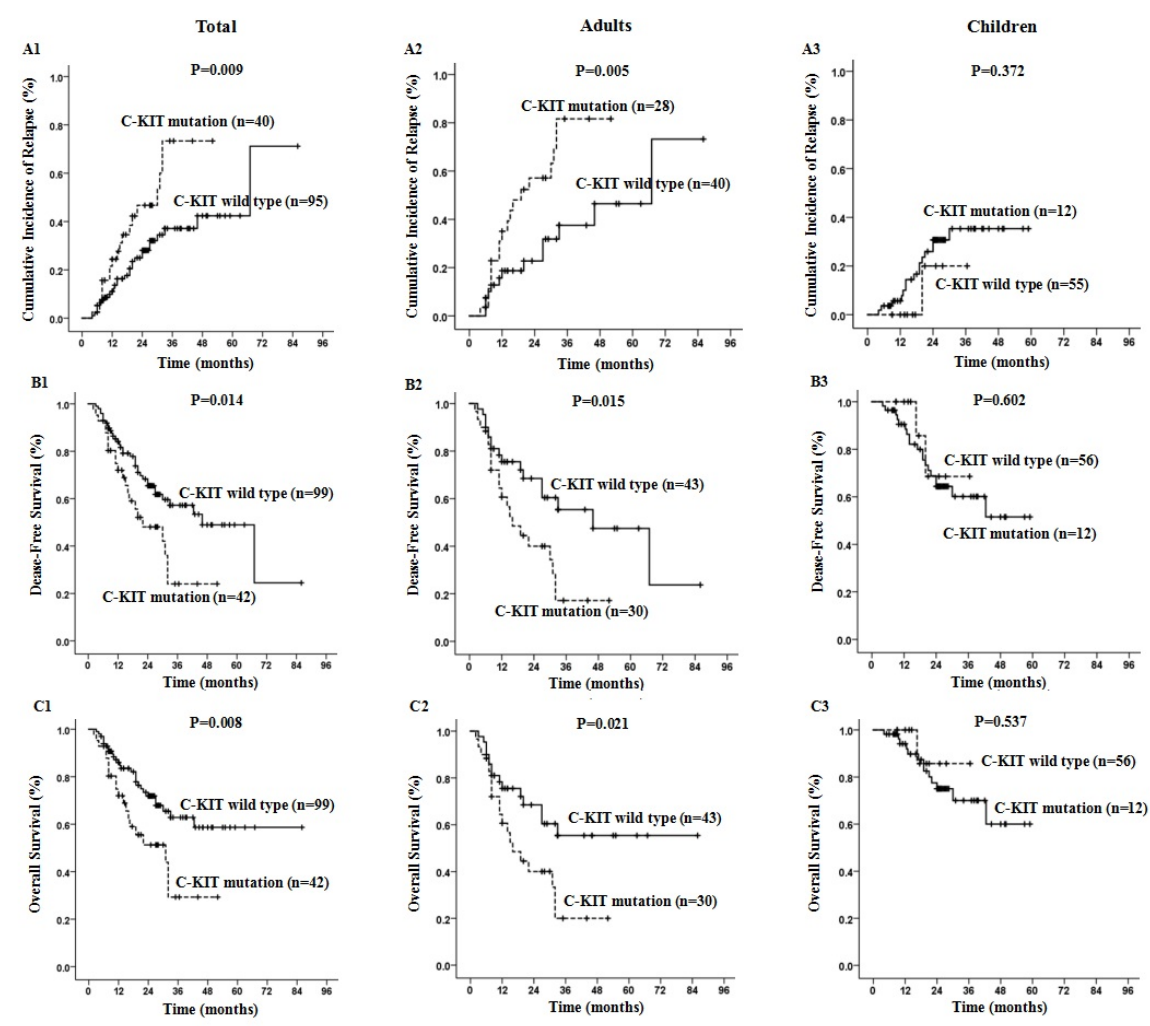

\section{Hosted file}

Table 1.pdf available at https://authorea.com/users/365426/articles/485548-aml1-etopositive-acute-myeloid-leukemia-presents-different-phenotype-in-pediatric-and-adultpatients

\section{Hosted file}

Table 2.pdf available at https://authorea.com/users/365426/articles/485548-aml1-etopositive-acute-myeloid-leukemia-presents-different-phenotype-in-pediatric-and-adultpatients

\section{Hosted file}

Table 3.pdf available at https://authorea.com/users/365426/articles/485548-aml1-etopositive-acute-myeloid-leukemia-presents-different-phenotype-in-pediatric-and-adultpatients 